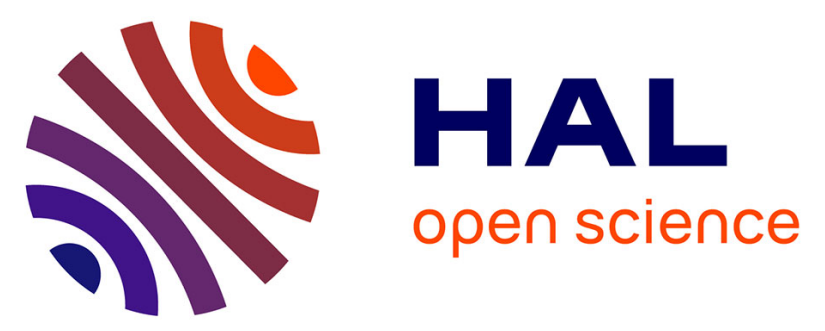

\title{
Modification of erbium photoluminescence decay rate due to ITO layers on thin films of SiO2:Er doped with Si-nanoclusters
}

\author{
M. Wojdak, H. Jayatilleka, M. Shah, A. J. J Kenyon, Fabrice Gourbilleau, \\ Richard Rizk
}

\section{To cite this version:}

M. Wojdak, H. Jayatilleka, M. Shah, A. J. J Kenyon, Fabrice Gourbilleau, et al.. Modification of erbium photoluminescence decay rate due to ITO layers on thin films of $\mathrm{SiO} 2$ : Er doped with Sinanoclusters. Journal of Luminescence, 2013, 136, pp.407. hal-00786018

\section{HAL Id: hal-00786018 https://hal.science/hal-00786018}

Submitted on 26 Jun 2018

HAL is a multi-disciplinary open access archive for the deposit and dissemination of scientific research documents, whether they are published or not. The documents may come from teaching and research institutions in France or abroad, or from public or private research centers.
L'archive ouverte pluridisciplinaire HAL, est destinée au dépôt et à la diffusion de documents scientifiques de niveau recherche, publiés ou non, émanant des établissements d'enseignement et de recherche français ou étrangers, des laboratoires publics ou privés. 


\title{
Modification of erbium photoluminescence decay rate due to ITO layers on thin films of $\mathrm{SiO}_{2}$ : Er doped with Si-nanoclusters
}

\author{
M. Wojdak ${ }^{\text {a,* }}$, H. Jayatilleka ${ }^{\text {a,b }}$, M. Shah ${ }^{\text {a }}$, A.J. Kenyon ${ }^{a}$, F. Gourbilleau ${ }^{c}$, R. Rizk ${ }^{c}$ \\ a Department of Electronic and Electrical Engineering, University College London, Torrington Place, London WC1E 7JE, United Kingdom \\ ${ }^{\mathrm{b}}$ Department of Electrical and Computer Engineering, University of Toronto, 10 King's College Road, Toronto, Ontario, Canada M5S 3G4

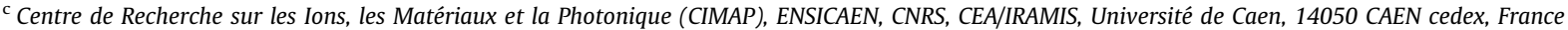

Keywords:

Photoluminescence, Erbium,

Silicon nanoclusters, Indium

tin oxide, Purcell effect.

\begin{abstract}
A B S T R A C T
During the fabrication of MOS light emitting devices, the thin film of active material is usually characterized by photoluminescence measurements before electrical contacts are deposited. However, the presence of a conductive contact layer can alter the luminescent properties of the active material. The local optical density of states changes due to the proximity of luminescent species to the interface with the conductive medium (the top electrode), and this modifies the radiative rate of luminescent centers within the active layer. In this paper we report enhancement of the observed erbium photoluminescence rate after deposition of indium tin oxide contacts on thin films of $\mathrm{SiO}_{2}$ : $\mathrm{Er}$ containing silicon nanoclusters, and relate this to Purcell enhancement of the erbium radiative rate.
\end{abstract}

\section{Introduction}

During preparation of electroluminescent devices, optimization of an active layer material is performed using photoluminescence (PL), amongst other characterization techniques. When the growth of the active film has been optimized, electrical contacts are deposited and electrical characteristics and electroluminescence are tested. However, the presence of the contacts can alter the luminescent properties of the active layer through the Purcell effect [1], in which the spontaneous emission rate of an emitter depends on its dielectric surroundings and the presence of interfaces between the media of different permittivities. This is described by variations in the local optical density of states (LDOS) [2,3]. Enhancement of the radiative rate of erbium in silica glass in the proximity of liquids with various refractive indices was previously demonstrated, hence confirming the effect [4]. Such an enhancement may also be expected in the proximity of an interface with metal [5]. The decay rate of a luminescent center near a dielectric-metal interface can be also modified by other processes like surface plasmon excitation or lossy surface wave excitation $[3,6]$. Using semimetallic transparent Indium Tin Oxide (ITO) contacts, it is possible to measure PL lifetimes and intensity through the contacts. We observe that the

\footnotetext{
* Corresponding author. Tel.: +44 207679 2162; fax: +44 2073889325.

E-mail addresses: m.wojdak@ucl.ac.uk (M. Wojdak), t.kenyon@ucl.ac.uk (A.J. Kenyon).
}

presence of ITO, which is extensively used as a transparent electrical contact in light emitting devices, can also increase the erbium PL decay rate. Importantly, the process is reversible by removing the ITO layer, confirming that the change is not due to diffusion of metal ions into the active layer, or chemical changes in the sample.

\section{Experimental}

Substoichiometric $\mathrm{SiO}_{x}$ : Er thin films were deposited by sputtering on $\left\langle\begin{array}{lll}1 & 0 & 0\end{array}\right\rangle$ p-type silicon wafers. Deposition details were reported in Ref. [7]. The silicon excess was 7 at\%, as estimated by FTIR measurements. The erbium concentration was estimated to be $2.2 \times 10^{20} \mathrm{~cm}^{-3}$, based on SIMS measurements of a similar sample. After deposition, the samples were annealed at $900{ }^{\circ} \mathrm{C}$ for $1 \mathrm{~h}$ under nitrogen flux in order to nucleate and grow silicon nanoclusters (Si-ncs). All the samples have the same material composition, but different thickness, which was determined by spectroscopic ellipsometry. ITO layers of thickness $60 \mathrm{~nm}$ were deposited by sputtering, and standard photolithography was carried out to form electrical contacts. PL measurements were performed using a Bentham M300 single grating monochromator and a NIR-sensitive Hamamatsu photomultiplier (R5509-72). The pumping of erbium photoluminescence in Si-ncs doped $\mathrm{SiO}_{2}: \mathrm{Er}$ can be realized in two modes: direct and indirect excitation. For direct excitation, we used a Spectra Diode Laboratories MOPA laser with wavelength $\lambda_{e x c}=980 \mathrm{~nm}$. The corresponding photon 
energy coincides with energy of second excited state of $\mathrm{Er}^{3+}$ ion, and incident photons can be absorbed directly by the ions. For indirect excitation, a DPSS laser emitting at wavelength $\lambda_{e x c}=473 \mathrm{~nm}$ was used. In this mode, incident photons are nonresonant with $\mathrm{Er}^{3+}$ energy levels, and the only available excitation path is absorption by Si nanoclusters and subsequent energy transfer to erbium ion [8]. In both cases laser beam was modulated with a Pockels cell. Time-resolved PL transients were recorded with a digital oscilloscope.

\section{Results and discussion}

Fig. 1 presents PL spectra of erbium emission obtained under $473 \mathrm{~nm}$ excitation, and the inset shows the evolution of PL lifetime with the thickness, as obtained with $473 \mathrm{~nm}$ and $980 \mathrm{~nm}$ excitations. Four samples were studied, with film thickness of $27 \mathrm{~nm}, 79 \mathrm{~nm}, 119 \mathrm{~nm}$, and $170 \mathrm{~nm}$. Although the material composition is intentionally identical for all samples, PL intensity does not depend linearly on the film thickness. This is due to the fact that difference in thickness leads to different stress in the samples, which affects nucleation and growth of Si-ncs during thermal treatment $[9,10]$. The inset shows time constants of PL decay. The PL transients for $980 \mathrm{~nm}$ excitation were fitted well with a single exponential decay function with lifetimes ranging from $2 \mathrm{~ms}$ to $4.2 \mathrm{~ms}$. The PL transients recorded with $473 \mathrm{~nm}$ excitation slightly diverge from single exponential behavior and better fits are obtained with double exponential decay function. The time constants of longer components are shown in the inset of Fig. 1, they range from $1.7 \mathrm{~ms}$ to $3 \mathrm{~ms}$, which is typical for indirectly pumped erbium $\mathrm{PL}$ in Si-ncs doped $\mathrm{SiO}_{2}$ :Er. In the indirect pumping mode we can excite only erbium ions located close to $\mathrm{Si}$ nanoclusters, as the energy transfer is limited to a certain distance [11]. Within this limit, erbium ions located at a longer distance from Si-nc contribute to slower PL decay, and those located closer to Si-nc contribute a faster PL decay due to enhancement of radiative lifetime in the proximity of Si nanoclusters $[12,13]$. This leads to characteristically double exponential PL transients. It should be noted that for indirect excitation the effective excitation cross section is few orders of magnitude higher than that for direct pumping, but only small fraction of erbium ions is coupled to Si nanoclusters [14]. The direct pumping is much less efficient, but can excite large number of ions

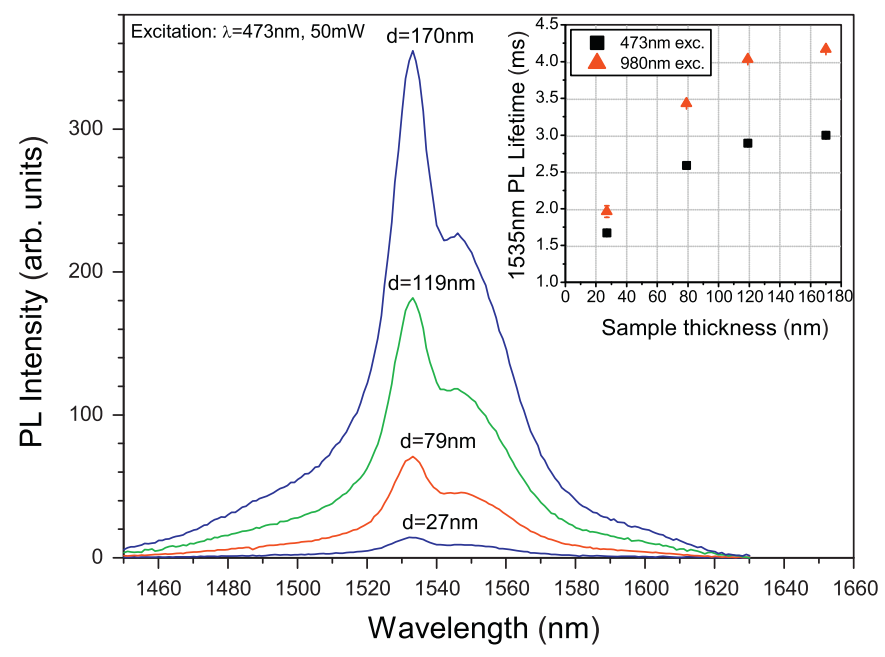

Fig. 1. Photoluminescence spectra obtained with indirect excitation and (in the inset) PL lifetimes of erbium luminescence obtained with direct $(980 \mathrm{~nm})$ and indirect $(473 \mathrm{~nm})$ excitation. including those non-coupled to Si-ncs. This leads to different PL decay characteristics in both modes.

Fig. 2 shows how PL transients are modified due to the presence of the ITO layer (for the sample of thickness $79 \mathrm{~nm}$ ). The PL transients are normalized and it is clear that PL decay rate is increased under the ITO layer. For direct pumping $(980 \mathrm{~nm})$ the PL transients remain single exponential under ITO for most of the samples. Only for the thinnest sample double exponential decay was observed, but the dominant longer component was still shorter compared to the PL lifetime recorded on an area without the ITO. For indirect pumping $(473 \mathrm{~nm})$ the PL decay rate is also increased under the ITO layer. In this case the PL transients remain double exponential and only the longer component is modified, the shorter component does not seem to be affected, which will be discussed later.

Fig. 3 shows the change in the observed PL decay time due to the presence of the ITO layer. The ratio of PL decay time under the ITO layer to that in the areas without the ITO is plotted as a function of the thickness of active layer. For the data where the double exponential function was used, the longer components are compared. For all the samples we observe an increase of erbium PL decay rate due to the ITO layer. In most cases there is also an increase of PL intensity. However, we observed that the power of reflected laser beam was lower when the laser illuminated areas covered by the ITO layer; in this case the ITO layer behaves as an antireflective coating and the effective pumping power

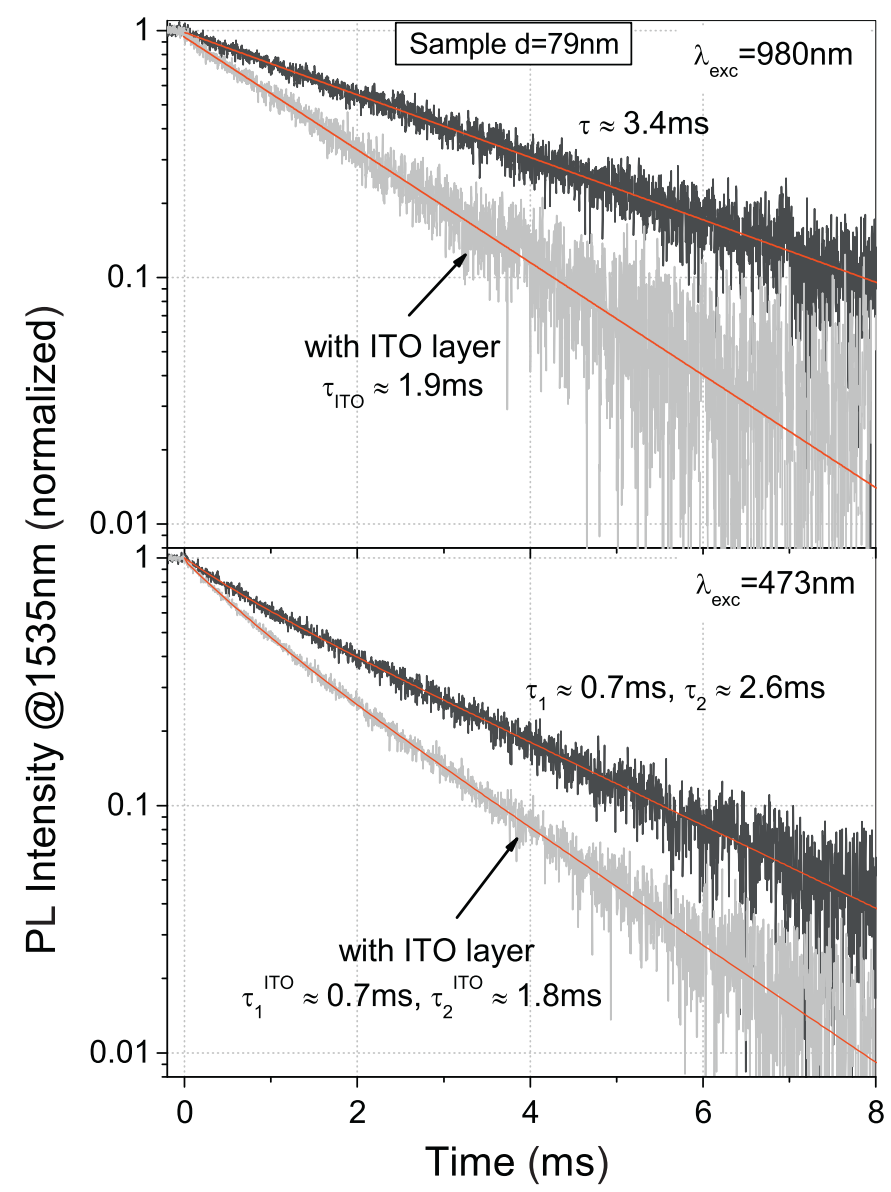

Fig. 2. Example of normalized photoluminescence transients in the areas of sample where there is no ITO (longer lifetimes) and covered with ITO (shorter lifetimes). For direct excitation $(980 \mathrm{~nm})$ the transients were fitted with single exponential decay function, and for indirect excitation $(473 \mathrm{~nm})$ double exponential decay function was used. 


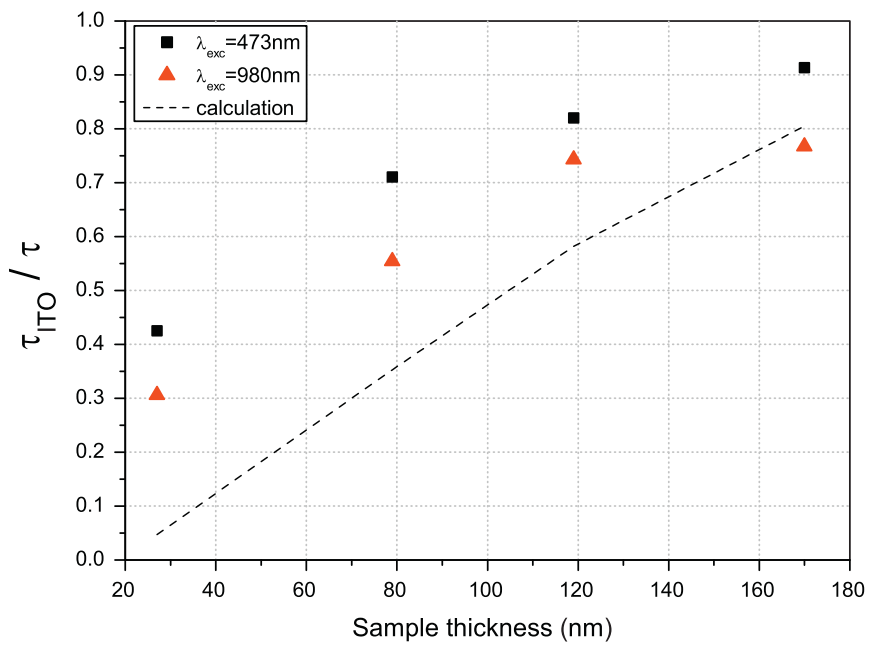

Fig. 3. Ratio of PL decay times with and without the ITO layers $\left(\tau_{\text {ITO }}\right.$-with ITO, $\tau$-without ITO). Experimental results obtained with indirect $(473 \mathrm{~nm})$ and direct $(980 \mathrm{~nm})$ excitations are compared with results of the theoretical calculation (dashed line).

inside the film may be higher, consequently PL intensities cannot be compared.

The rate of spontaneous emission from a dipole in a slab structure can be investigated theoretically by calculating the emitted power from the dipole while taking into account the reflections from the discontinuities in the structure [15]. We simulated the enhancement of decay rates of $\mathrm{Er}^{3+}$ due to the presence of the ITO and silicon surfaces using this method. The decay rate $(w)$, normalized with respect to the decay rate of a dipole located far from any interface in the homogenous medium $\left(w_{\text {bulk }}\right)$ is obtained by integrating the classical dissipated power $(P)$ over the in-plane wave vector $(p)$ :

$\frac{w}{w_{\text {bulk }}}=1-\eta_{\text {bulk }}+\eta_{\text {bulk }} \int_{0}^{\infty} \frac{1}{P_{\text {bulk }}} d p \frac{d P}{d p}$

Here, $\eta_{\text {bulk }}$ is the quantum efficiency of the dipole and $P_{b u l k}$ is the power dissipated from the dipole in the initial bulk homogenous media. The integrand in the above equation can be calculated by considering the Fresnel reflections for any number of surfaces near the dipoles $[15,16]$.

Erbium in our samples is contained within the thin $\mathrm{SiO}_{2}$ layers doped with Si-ncs. Therefore, we first estimate the quantum efficiency $\eta_{\text {bulk }}$ of erbium in the bulk $\mathrm{SiO}_{2}$ doped with $\mathrm{Si}$ nanoclusters. If the bulk homogenous medium had no defects, then the quantum efficiency of erbium in this medium would be $\eta_{0}=1$. The radiative decay rate in this case is estimated to be $w_{0}^{\text {rad }}=70 \mathrm{~s}^{-1}$ [17]. Now, we assume that the defects in the media would increase the decay rate by providing additional non-radiative decay paths and that the radiative decay rate $\left(w_{0}^{\text {rad }}\right)$ would remain the same. With this assumption, the quantum efficiency of erbium in bulk $\mathrm{SiO}_{2}$ doped with Si-ncs can be written as $\eta_{\text {bulk }}=w_{0}^{\text {rad }} / w_{\text {bulk }}$. We use Eq. (1) in our reference structures without ITO layers and obtain the following equation for $\eta_{\text {bulk }}[17,18]$ :

$\eta_{\text {bulk }}=\frac{1}{1+w / w_{0}^{\text {rad }}-\int_{0}^{\infty}\left(1 / P_{\text {bulk }}\right) d p(d P / d p)}$

In Eq. (2), we can determine $w$ from the PL measurements. The integrand is theoretically calculated using the Fresnel reflections on our reference structures with thin layers (of known thicknesses) of erbium and $\mathrm{Si}$-nc doped $\mathrm{SiO}_{2}$ on silicon substrates. From this calculation we obtain $\eta_{\text {bulk }}$ values of $0.2075,0.2878$, 0.3185 , and 0.3190 for the samples of thickness $27 \mathrm{~nm}, 79 \mathrm{~nm}$, $119 \mathrm{~nm}$, and $170 \mathrm{~nm}$, respectively.
The above calculated bulk quantum efficiencies $\eta_{\text {bulk }}$ can be used in Eq. (1) to evaluate the decay rate enhancement due to presence of interfaces. We consider two systems: the first one is a thin film on Si-substrate (enhancement $w / w_{\text {bulk }}$ ), which was already used to determine $\eta_{\text {bulk }}$ values, the second one is a thin film on a Sisubstrate and with the ITO layer on the top (enhancement $w_{\text {ITO }}$ $\left.w_{\text {bulk }}\right)$. In both cases, Eq. (1) gives enhancements with respect to $w_{\text {bulk }}$, but the ratio of these two results gives ratio of decay rate for the thin films with or without ITO $\left(w_{\text {Iто }} / w\right)$. In this way, we can predict the rate enhancement due to the ITO layer and compare it with the experimental results. In our calculations, we have assumed the erbium in Si-nc-doped $\mathrm{SiO}_{2}$ to be arbitrarily oriented and evenly distributed within the oxide matrix with a spacing of $5 \mathrm{~nm}$ between the ITO/ $\mathrm{SiO}_{2}$ interface and the nearest erbium ion $[17,19,20]$. The thickness of the ITO layers deposited is $60 \mathrm{~nm}$ and the refractive index of ITO at $1535 \mathrm{~nm}$ wavelength is considered to be $(0.5+0.4 i)$ [21]. The refractive index of Si-nc-doped $\mathrm{SiO}_{2}$ at the same wavelength is 1.5 [16].

In Fig. 3, the results of the above calculation (dashed line) are compared with the corresponding experimental values of the ratio of decay times $\left(\tau_{\text {Iто }} / \tau\right)$ with and without the ITO layer for both direct and indirect pumping.

For most samples the calculation predicts enhancement higher than that observed. A possible source of error is the optical constants of our ITO being different from that reported in literature, as they are sensitive to deposition conditions. However, perhaps more significantly, we note that the actual distribution of erbium ions and Si nanoclusters within the layer is unknown. In our calculation we assumed an uniform distribution. This should be correct for experiment with direct pumping $(980 \mathrm{~nm})$ which excites all erbium ions in the layer. However, it may be inaccurate for indirect mode-we note that the region of the layer close to the silicon substrate may be depleted of silicon nanoclusters due to interfacial stress [10]. But the real distribution of Si-ncs is not known, and using uniform distribution in the calculations is only approximation. Nevertheless, the general form of the variation in radiative enhancement with sample thickness is similar between the predication and experimental plots.

The observed radiative rate enhancement is also more pronounced with direct excitation $(980 \mathrm{~nm})$ than with indirect pumping $(473 \mathrm{~nm})$. Here we note that with indirect excitation $(473 \mathrm{~nm})$ we excite only erbium ions close to $\mathrm{Si}$ nanoclusters. Their radiative rate is already increased due to the proximity of Si-nc $[12,13]$ and further modification of total decay rate by the effects caused by the more distant ITO layer is smaller. In other words, the effect of ITO on the top of the thin film can be screened by Si nanoclusters. For the same reason, the shorter component in double exponential transients is not modified by the presence of ITO, as it originates from erbium ions located very close to Si-ncs. On the other hand, the situation for indirect excitation can be more complicated. It is not clear whether changing LDOS due to the proximity of the conductive medium can also modify the excitation transfer rate from Si nanoclusters to erbium. While it has been reported that modification of the transfer rate occurs [22], there are also reports that the transfer rate does not depend on LDOS $[23,24]$. This remains controversial, and on the basis of our measurements we are not able to determine whether there is any enhancement of transfer rate in addition to that of the radiative rate.

\section{Conclusions}

We report here that presence of ITO top electrodes strongly modifies the erbium luminescence decay rate in a similar way to metallic overlayers or higher refractive index media. The effect is 
weaker with indirect excitation, probably due to the fact that erbium ions coupled to Si nanoclusters have already modified radiative rate due to the proximity of Si-nc and additional modification by ITO layer is weaker. Comparison with theoretical calculations gives only qualitative agreement. The observed effect can be a combination of radiative enhancement, interaction with surface plasmons, and lossy surface wave excitation, and is likely to be at least partly responsible for the short luminescence lifetime observed for electroluminescence from $\mathrm{SiO}_{x}$ : Er devices [25].

\section{References}

[1] E.M. Purcell, Phys. Rev. 69 (1946) 681

[2] R. Sprik, B.A. van Tiggelen, A. Lagendijk, Europhys. Lett. 35 (1996) 265.

[3] J. Kalkman, H. Gersen, L. Kuipers, A. Polman, Phys. Rev. B 73 (2006) 075317.

[4] E. Snoeks, A. Lagendijk, A. Polman, Phys. Rev. Lett. 74 (1995) 2459.

[5] K.H. Drexhage, J. Lumin. 1-2 (1970) 693.

[6] W.L. Barnes, J. Mod. Opt. 45 (1998) 661.

[7] K. Hijazi, R. Rizk, J. Cardin, L. Khomenkova, F. Gourbilleau, J. Appl. Phys. 106 (2009) 024311

[8] A.J. Kenyon, P.F. Trwoga, M. Federighi, C.W. Pitt, J. Phys.: Condens. Matter 6 (1994) L319.
[9] I. Ahmad, M.P. Temple, A. Kallis, M. Wojdak, C.J. Oton, D. Barbier, H. Saleh, A.J. Kenyon, W.H. Loh, J. Appl. Phys. 104 (2008) 123108

[10] S. Cueff, C. Labbe, O. Jambois, B. Garrido, X. Portier, R. Rizk, Nanoscale Res. Lett. 6 (2011) 395.

[11] Ji-Hong Jhe, Jung H. Shin, Kyung Joong Kim, Dae Won Moon, Appl. Phys. Lett. 82 (2003) 4489.

[12] V.V. Klimov, Phys. Usp. 46 (2003) 979

[13] P. Horak, W.H. Loh, A.J. Kenyon, Opt. Express 17 (2009) 906.

[14] M. Wojdak, M. Klik, M. Forcales, O.B. Gusev, T. Gregorkiewicz, D. Pacifici, G. Franzò, F. Priolo, F. Iacona, Phys. Rev. B 69 (2004) 233315.

[15] G.W. Ford, W.H. Weber, Phys. Rep. 113 (1984) 195.

[16] A. Hryciw, Y.C. Jun, M.L. Brongersma, Opt. Express 17 (2009) 185.

[17] J. Bao, N. Yu, F. Capasso, T. Mates, M. Troccoli, A. Belyanin, Appl. Phys. Lett. 91 (2007) 131103.

[18] X. Brokmann, L. Coolen, M. Dahan, J.P. Hermier, Phys. Rev. Lett. 93 (2004) 107403.

[19] P.T. Worthing, R.M. Amos, W.L. Barnes, Phys. Rev. A 59 (1999) 865

[20] H. Jayatilleka, A. Nasrollahy-Shiraz, A.J. Kenyon, Opt. Express 19 (2011) 24569.

[21] R.A. Synowicki, Thin Solid Films 313-314 (1998) 394.

[22] T. Nakamura, M. Fujii, K. Imakita, S. Hayashi, Phys. Rev. B 72 (2005) 235412.

[23] M.J.A. de Dood, J. Knoester, A. Tip, A. Polman, Phys. Rev. B 71 (2005) 115102

[24] S. Miura, T. Nakamura, M. Fujii, M. Inui, S. Hayashi, Phys. Rev. B 73 (2006) 245333.

[25] O. Jambois, F. Gourbilleau, A.J. Kenyon, J. Montserrat, R. Rizk, B. Garrido, Opt. Express 18 (2010) 2230 